\title{
An Investigation of Two Stereotypes Regarding the Writing of Engineers
}

Sharon Kowalski

NAWCTSD

Orlando, FL

sharon_kowalski@ntsc.navy.mil
John Oriel

Naval Air Warfare Center Training Systems Division

Orlando, FL 32826-3224

john_oriel@ntsc.navy.mil

This statistical study makes use of data gathered during quality-control inspections to examine the validity of two commonly held stereotypes. The first of these is the belief that documents containing superficial errors are likely to be flawed in a more substantial manner; the second is that engineers tend to be especially inept as technical writers. In both cases, the data indicate that the stereotypes are invalid.

\section{Introduction}

Technical communicators often spend a great portion of their careers painstakingly editing text that was drafted by engineers. This labor is customarily deemed necessary because most people tend to believe that works containing superficial errors are likely to be flawed in more substantive ways, such as conveying incorrect information or faulty opinions. So much exposure to errors made by engineers has led many technical communicators to believe that engineers are especially inept as writers. However, the evidence offered in support of that opinion, convincing as it may seem, is usually anecdotal. Literature that cites deficits in the communication skills of other types of professionals (Sullivan 1996), even medical doctors (Heymont 1996), also raises questions about whether or not engineers appear to be such poor writers when viewed from a broader perspective. The purpose of this study was to examine some quantitative evidence regarding the validity of these two common stereotypes.

\section{Data gathering}

The data were gathered as part of a quality-management effort. More specifically, they were records of the number and types of errors made by adult workers in the preparation of two types of documents known as specifications and statements of work. The entire text of 63 documents was examined, about half of which had been written by engineers and half by non-engineers, most of whom were social-science professionals. The entire corpus amounted to about 1,200 single-spaced, typed pages of text. Both types of documents, which are prepared for inclusion in contracts, are subject to standardization in format and content, and are usually subject to careful editing and correction before release. "Statement of work" is a bureaucratic term used to distinguish between specifications for services versus specifications for goods. The Grovernment rigorously separates the two types of specifications for practical reasons, the primary one being to facilitate the enforcement of a statutory ban on contracting for personal services, which are required by law in most cases to be performed only by Federal employees. 
The content and format for specifications is standardized by MIL-STD-961D, and for statements of work the applicable standard is MIL-HDBK-245D. Both documents dictate an outline that may be tailored to specific cases, but in general must be followed as closely as practicable. Both standards provide a great deal of guidance needed in order that authors may easily comply with numerous laws and regulations relating to Government purchasing. The authors, therefore, need not perform an audience analysis or design the document. What is expected of them is that they organize and present the contractual requirements in accordance with the detailed instructions set forth by the two military standards and by the normal conventions of clarity and correctness common to all forms of business writing. Violations of those standards were considered errors for the purpose of our data gathering, judged by personnel known to be both skilled and experienced at inspecting the types of documents under study. Errors marked by the inspectors were categorized in a manner similar to that described in an earlier report (Oriel 1993), and logged to produce the data.

\section{Analysis}

In keeping with common belief, we hypothesized that the frequency of superficial errors ought to be related to the frequency of the more serious, substantive errors. Because of the especially sensitive nature of specifications, such documents require particular attention to many details that one would ordinarily consider superficial. Ideally, one would like to call "substantive" all errors that might affect the meaning or comprehensibility of the text, and all the rest "superficial." For practical reasons, however, we had to choose a more tangible set of criteria. One hope in doing so was that an easily monitored variable could be identified that would permit clerical personnel to quickly estimate the number of serious errors likely to be found in the course of a thorough inspection. Hence, the only types of errors categorized as superficial were the following:

- spelling errors,

- capitalization errors,

- punctuation errors,

- spacing errors, and

- paragraph numbering errors.

Even with so strict an interpretation of the word "superficial," there is still a slight probability that a few substantive errors might be categorized incorrectly according to the ideal. When reviewing specifications, one occasionally finds errors of the types listed above that could contribute to a misunderstanding: witness words like "because," which may introduce either a restrictive or nonrestrictive dependent clause, depending on whether or not they are preceded by a comma (Sabin 1993). In such cases, a missing comma can have a substantial effect on meaning. Even though we look very carefully for such errors, we have found that their relative frequency is quite small, and the few that we found ought not to contribute a significant amount of error to our study's results. 
The errors categorized as substantive are of the following types:

- inconsistency of meaning;

- lack of clarity;

- misstatement of requirements;

- singular where plural was appropriate, or vice versa;

- violation of a law or regulation;

- incorrectly specified numerical values or units;

- agreements to agree at some future time, a type of contractual faux pas;

- warranting suitability of an item for a specific purpose;

- unnecessarily specifying a certain vendor or part;

- specifying design details instead of performance requirements;

- omitted, outdated, or unnecessary information; and

- errors in word usage, primarily "shall," "will," "any," "as required," "up to," "or," and "and/or."

Regarding the probabilities of error detection, all 63 documents were inspected for superficial errors by the same inspector, an experienced executive assistant, so we feel confident that they were all evaluated equally. Most of the substantive inspections were divided evenly between two individuals whose detection rates average about the same. Only four documents were inspected by others during periods of excessive workload.

The figures reported here were prepared by adding the numbers of errors logged in each of the categories listed and normalizing the totals by dividing the total by the number of pages in the document. To complete the test of the first hypothesis, the correlation coefficient for the resulting set of error-frequency data was computed. Only a slight positive correlation, .23 , was found between the frequencies of the superficial errors and that of the more substantive ones, certainly not a relationship that could be useful as a predictor. Intercorrelations were also computed on all the individual error categories, and no other practically significant relationships were found.

Since the authorship of the documents was divided about evenly between engineers and non-engineers, the data provided an opportunity to compare the rates at which the two groups made errors, and thus gauge some aspects of their relative achievement levels as technical writers. The hypothesis set forth by the stereotype in this case would be that the work of the engineers tends to contain more errors than that of the non-engineers, and one can clearly see in the histogram, Figure 1, that such is not true. 


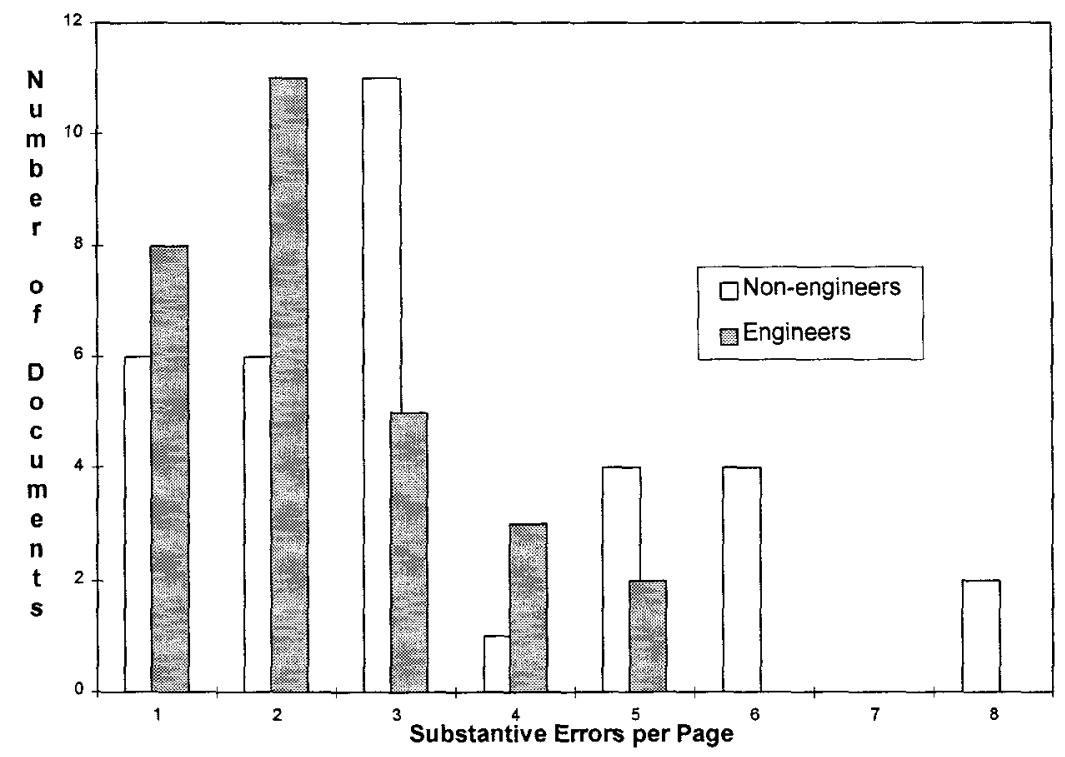

Figure 1. Frequencies of Substantive Errors for Engineers and Non-engineers

The mean numbers of errors per page, 1.8 for the engineers and 3.0 for the nonengineers, and the standard deviations, 1.1 and 1.8 respectively, indicate that the engineers' work tended to have slightly fewer errors and that the error rates were less broadly distributed for the engineers as a group. On the whole, however, the distributions were quite similar. As a point of reference, Gilb and Graham (1993) indicate that software specifications inspected to their own more rigorous criteria often have between five and twenty errors per page.

A more formal test of the hypothesis can be done by referring to Tchebychev's Theorem, which applies to all sets of measurements, regardless of whether or not their distribution follows the normal bell-shaped curve, and which indicates that $75 \%$ of all measurements should lie within two standard deviations of the mean (Mendenhall 1971). The data indicate that $40 \%$ of the documents prepared by the non-engineers had error rates greater than two standard deviations above the mean for the work of the engineers. We conclude, then, on the basis of the $15 \%$ excess, that there is a statistically significant difference between the distributions of the error rates.

\section{Conclusion}

Even though we have found that the number of superficial errors is not a useful indicator of the relative quality of a document, we cannot subscribe to the notion that such errors should be ignored by editors or that students need not be trained to recognize and correct them. Engineering documents, particularly specifications, should be made as correct as humanly possible. A few minutes' effort spent in making a document right will pay off many fold by its effect in enhancing credibility and authoritativeness.

Regarding the comparison between the engineers and non-engineers, we can confidently say that the stereotype of engineers as especially inept writers is invalid for the situation examined. Certainly, the strictly defined nature of the documents involved in the study 
eliminates many important technical writing skills as variables, and hence, one cannot consider our results to make a meaningful statement about engineers as technical writers. However, it should be taken as a clear indication that technical communicators ought not to criticize engineers as a group, since their writing does not necessarily contain more errors than that of other professionals.

\section{References}

Gilb, T. and Graham, D. Software Inspection. Reading, MA: Adison Wesley, 1993.

Heymont, George. "Dysfunctional Doctors in Denial" Keeping Abreast of Medical

Transcription. Jan. 1997 http://www.wwma.com/kamt/kamt006.html (2 Apr. 1997)

Mendenhall, William and Reinmuth, James E. Statistics for Management and Economics. Belmont, CA: Duxbury Press, 1971.

Oriel, John T. Engineering Specification Editing Tools, NAWCTSD, Technical Report 93-022. Orlando: Naval Air Warfare Center Training Systems Division, 1993.

Sabin, William A. The Gregg Reference Manual, seventh ed. New York: Glencoe Div, Macmillan/McGraw-Hill 1993.

Sullivan, Tim. "Has School Reform Flunked?” World and I, Sept. 1996.

http://www.worldandi.com/archive/cisep.htm (9 Apr. 1997) 